\title{
Anticoagulation in children: personalized strategies
}

\author{
"In the future, anticoagulation in children may also be \\ individualized with the use of pharmacogenetics ... \\ to identify patients who are at risk for increased toxicity or decreased \\ response to a medication using genetic information."
}

From a public health view, venous thromboembolism (VTE) is primarily a disease of the aging population with 100,000 deaths per year attributed to VTE. The US Surgeon General has recently issued a call to action concerning VTE risk to encourage public awareness and increased research on the causes, prevention and treatment of these conditions [101]. Although children are much less likely to have VTE, it is still an important issue particularly for children who are hospitalized with complex medical conditions. Traditionally, anticoagulation in children has been based on extrapolation from adult data and treatment guidelines. There is significant room for improvement in the evidence-based management of pediatric VTE.

“The thrombophilia evaluation has generally been utilized to explain the etiology of VTE and to predict the risk of VTE in family members, but it would be more useful if it could be used to modify therapy and improve clinical outcomes."

With the discovery of genetic risk factors for VTE occurrence and outcome (inherited thrombophilia) and the development of pharmacogenetic algorithms for warfarin, the future of pediatric anticoagulation should involve risk-based anticoagulation strategies and pharmacogenetic-based dosing regimens.

\section{Inherited thrombophilia \& risk-based anticoagulation}

In children, the vast majority of VTE is secondary to acquired risk factors such as central venous catheters, congenital heart disease or systematic inflammatory disorders [1]. However, inherited thrombophilia may also predispose to VTE in children, most often in combination with an acquired risk factor. Inherited thrombophilia was first used to describe members of a family with increased prevalence of VTE and a deficiency of antithrombin, and multiple inherited thrombophilia traits have subsequently been identified [2].

The Subcommittee for Perinatal and Pediatric Thrombosis of the Scientific and Standardization Committee of the International Society of Thrombosis and Haemostasis recommends a step-wise thrombophilia evaluation for children with VTE [3]. When thrombophilia testing has been performed in children with VTE, the results have been variable, $13-79 \%$ have been found to have inherited thrombophilia with the highest prevalence in older children [4-6]. The thrombophilia evaluation has generally been utilized to explain the etiology of VTE and to predict the risk of VTE in family members, but it would be more useful if it could be used to modify therapy and improve clinical outcomes.

One of the primary clinical outcomes of VTE is recurrence. The recurrence rate in children has been estimated at approximately 3 and $8 \%$ in neonates and children, respectively, but some investigators have reported rates as high as $21 \%$ in children with inherited thrombophilia [7-9]. More recently, a systematic review of inherited thrombophilia and thromboembolism in children indicated an overall rate of recurrence at $11.4 \%$ with a higher risk of recurrence associated with adolescence and discontinuation of anticoagulation. Of interest, the recurrence rate was also significantly higher with most inherited thrombophilia traits [9].

\section{Decision making in children with inherited thrombophilia}

In general, children with secondary VTE are anticoagulated for 3 months and children with idiopathic VTE are anticoagulated for 6-12 months. In the absence of definitive data, clinicians have developed recommendations for patients who might warrant longer or indefinite
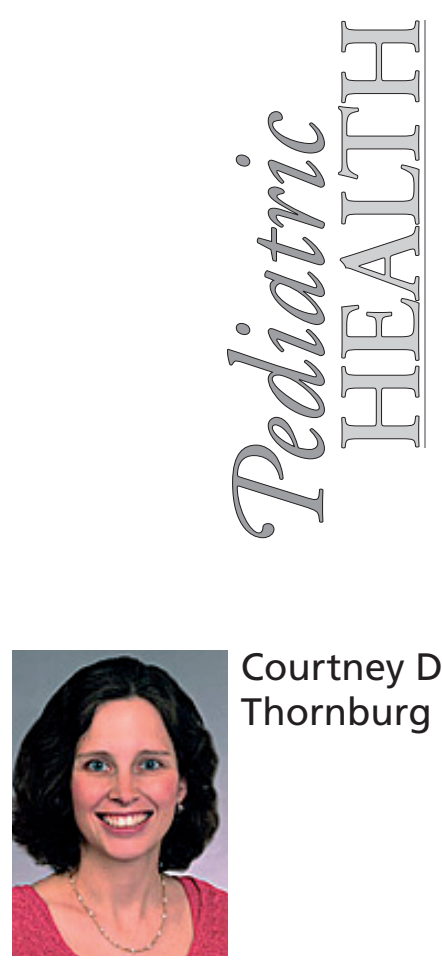

Duke University Medical Center, Durham, NC 27710, USA

Tel.: +19196843401

Fax: +19196817950

thorn006@mc.duke.edu

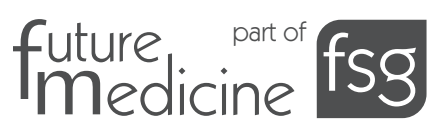


anticoagulation. Manco-Johnson incorporated inherited thrombophilia traits into a model for determining the duration of anticoagulant therapy in children [10]. Nowak-Göttl has also proposed an anticoagulation strategy that incorporates inherited traits [11]. In adult studies, thrombophilia testing has not been demonstrated to reduce the risk of VTE recurrence, but a randomized, controlled trial of standard anticoagulation versus risk-based anticoagulation has not been completed [12]. Hopefully, future studies will identify patients who need prolonged therapy for optimal outcome and identify those who will still have good outcome even with shortened therapy. Pediatric-specific studies are warranted since the potential benefits of prolonged anticoagulation must be balanced with risk of bleeding associated with decades of anticoagulation.

"Children who require long-term anticoagulation could benefit from an individualized approach that improves the safety and efficacy of warfarin."

Regardless of why thrombophilia testing is carried out, medical providers must consider the potential impact on asymptomatic family members [13]. Pediatric hematologists are often asked to evaluate children with a family history of inherited thrombophilia. Such testing is controversial since there are there are no standard treatment or thromboprophylaxis guidelines for children or adults who have inherited thrombophilia but have not had VTE. The problems with testing include potential for misinterpretation of results by practitioners and family, expense, concerns regarding insurance discrimination and the potential impact on both contraceptive options and future childbearing plans.

\section{Warfarin pharmacogenetics \& individualized anticoagulation}

In the future, anticoagulation in children may also be individualized with the use of pharmacogenetics. The goal of pharmacogenetics is to identify patients who are at risk for increased toxicity or decreased response to a medication using genetic information.

Warfarin is the primary anticoagulant used for long-term anticoagulation in children, particularly those with congenital heart disease, antiphospholipid antibody syndrome and recurrent VTE. Dosing is empiric and derived from adult guidelines [14]. An algorithm to predict an individual's therapeutic dose is very attractive given the interindividual variation in dose response and a narrow therapeutic range. Some variation in warfarin response is explained by clinical factors. However, we now know that genetic variation in two key enzymes involved in warfarin pharmacokinetics affects the dose-response. Algorithms have been developed and tested in adults that incorporate both clinical and pharmacogenetic factors; these algorithms predict more than 50\% of the dose variation [15]. Although ongoing randomized, controlled trials are still investigating the impact on clinical outcome, Wadelius demonstrated that warfarin guided by pharmacogenetics should improve clinical outcomes in adults [16]. Research efforts are underway to develop pediatric-specific algorithms.

Before pharmacogenetic testing becomes widespread, the following questions should be addressed:

- Who should order the test?

- What type of counseling should be provided regarding implications for the family, expense, concerns about insurance discrimination and ancillary information that may be discovered as a result of testing [17]?

- How and where should the results be reported?

- Who should have access to the results?

In summary, clinical trial data are urgently needed to test the hypothesis that inherited thrombophilia testing in children can be incorporated into risk-based therapy and improve clinical outcome. In addition, pharmacogenetic strategies to improve warfarin anticoagulation should be studied in children. Children who require long-term anticoagulation could benefit from an individualized approach that improves the safety and efficacy of warfarin. The concepts of inherited risk-based therapy and pharmacogenetic-based medication dosing will likely be relevant to many chronic pediatric illnesses in the future. Pediatricians and pediatric subspecialists will need to determine how to incorporate this testing into clinical care.

\section{Financial \& competing interests disclosure}

The author has no relevant affliations or financial involvement with any organization or entity with a financial interest in or financial conflict with the subject matter or materials discussed in the manuscript. This includes employment, consultancies, honoraria, stock ownership or options, expert testimony, grants or patents received or pending, or royalties.

No writing assistance was utilized in the production of this manuscript. 


\section{Bibliography}

1. Kuhle S, Massicotte P, Chan A, Adams M, Abdolell M, de Veber G et al.: Systemic thromboembolism in children. Data from the 1-800-NO-CLOTS Consultation Service. Thromb. Haemost. 92(4), 722-728 (2004).

2. Nowak-Göttl U, Kurnik K, Krumpel A, Stoll M: Thrombophilia in the young. Hamostaseologie 28(1), 16-20 (2008).

3. Manco-Johnson MJ, Grabowski EF, Hellgreen M et al.: Laboratory testing for thrombophilia in pediatric patients. On behalf of the Subcommittee for Perinatal and Pediatric Thrombosis of the Scientific and Standardization Committee of the International Society of Thrombosis and Haemostasis (ISTH). Thromb. Haemost. 88(1), 155-156 (2002).

4. Ehrenforth S, Junker R, Koch HG et al: Multicentre evaluation of combined prothrombotic defects associated with thrombophilia in childhood. Childhood Thrombophilia Study Group. Eur. J. Pediatr. 158 (Suppl. 3), S97-S104 (1999).

5. Revel-Vilk S, Chan A, Bauman M, Massicotte P: Prothrombotic conditions in an unselected cohort of children with venous thromboembolic disease. J. Thromb. Haemost. 1(5), 915-921 (2003).

6. Revel-Vilk S, Kenet G: Thrombophilia in children with venous thromboembolic disease. Thromb. Res. 118(1), 59-65 (2006).
7. Nowak-Göttl U, Junker R, Kreuz W et al.: Risk of recurrent venous thrombosis in children with combined prothrombotic risk factors. Blood 97(4), 858-862 (2001).

8. Monagle P, Adams M, Mahoney M et al.: Outcome of pediatric thromboembolic disease: a report from the Canadian Childhood Thrombophilia Registry. Pediatr. Res. 47(6), 763-766 (2000)

9. Young G, Albisetti M, Bonduel M et al:: Impact of inherited thrombophilia on venous thromboembolism in children: a systematic review and meta-analysis of observational studies. Circulation 118(13), 1373-1382 (2008).

10. Manco-Johnson MJ: How I treat venous thrombosis in children. Blood 107(1), 21-29 (2006).

11. Nowak-Gottl U, Kurnik K, Krumpel A, Stoll M: Thrombophilia in the young. Hamostaseologie 28(1-2), 16-20 (2008).

12. Coppens M, Reijnders JH, Middeldorp S, Doggen CJ, Rosendaal FR: Testing for inherited thrombophilia does not reduce the recurrence of venous thrombosis. J. Thromb. Haemost. 6(9), 1474-1477 (2008).

13. Thornburg CD, Dixon N, Paulyson-Nunez K, Ortel T: Thrombophilia screening in asymptomatic children. Thromb. Res. 121(5), 597-604 (2008).

14. Monagle P, Chalmers E, Chan A et al: Antithrombotic therapy in neonates and children: American College of Chest
Physicians evidence-based clinical practice guidelines (8th edition). Chest 133(Suppl. 6), 887S-968S (2008).

15. Gage BF, Eby C, Johnson JA et al:: Use of pharmacogenetic and clinical factors to predict the therapeutic dose of warfarin. Clin. Pharmacol. Ther. 84(3), 326-331 (2008).

16. Wadelius M, Chen LY, Lindh JD et al.: The largest prospective warfarin-treated cohort supports genetic forecasting. Blood 113(4), 784-792 (2009).

17. Haga SB, Burke W: Pharmacogenetic testing: not as simple as it seems. Genet. Med. 10(6), 391-395 (2008).

\section{Website}

101. Office of the Surgeon General. Acting Surgeon General issues call to action to prevent deep vein thrombosis and pulmonary embolism.

www.surgeongeneral.gov/news/pressreleases/ pr20080915.htm

(Accessed December 2008).

\section{Affiliation}

- Courtney D Thornburg, MD, MS Duke University Medical Center, Durham, NC 27710, USA

Tel.: +1 9196843401

Fax: +1 9196817950

thorn006@mc.duke.edu 